\title{
The influence of communicative relations on facial responses to pain: Does it matter who is watching?
}

\author{
Anna J Karmann MSc, Stefan Lautenbacher PhD, Florian Bauer MSc, Miriam Kunz PhD
}

AJ Karmann, S Lautenbacher, F Bauer, M Kunz. The influence of communicative relations on facial responses to pain: Does it matter who is watching? Pain Res Manag 2014;19(1):15-22.

BACKGROUND: Facial responses to pain are believed to be an act of communication and, as such, are likely to be affected by the relationship between sender and receiver.

OBJECTIVES: To investigate this effect by examining the impact that variations in communicative relations (from being alone to being with an intimate other) have on the elements of the facial language used to communicate pain (types of facial responses), and on the degree of facial expressiveness.

METHODS: Facial responses of 126 healthy participants to phasic heat pain were assessed in three different social situations: alone, but aware of video recording; in the presence of an experimenter; and in the presence of an intimate other. Furthermore, pain catastrophizing and sex (of participant and experimenter) were considered as additional influences.

RESULTS: Whereas similar types of facial responses were elicited independent of the relationship between sender and observer, the degree of facial expressiveness varied significantly, with increased expressiveness occurring in the presence of the partner. Interestingly, being with an experimenter decreased facial expressiveness only in women. Pain catastrophizing and the sex of the experimenter exhibited no substantial influence on facial responses.

CONCLUSION: Variations in communicative relations had no effect on the elements of the facial pain language. The degree of facial expressiveness, however, was adapted to the relationship between sender and observer. Individuals suppressed their facial communication of pain toward unfamiliar persons, whereas they overtly displayed it in the presence of an intimate other. Furthermore, when confronted with an unfamiliar person, different situational demands appeared to apply for both sexes.

Key Words: Communicative relations; Facial expression; FACS; Pain; Social variations

Communication of pain can be defined as 'action', 'interaction' and 'transaction' (1). Whereas the 'action' can be considered to be the individual propensity or drive to display pain by overt behaviour, the 'interaction' and the 'transaction' both require the consideration of a receiver, who either only decodes the behavioural pain message or thereafter reacts to the sender, respectively. If 'interaction' or 'transaction' are the behavioural goals, the behavioural display of pain is likely subject to the relationship between the sender and receiver. Although facial responses represent one of the most prominent and informative nonverbal communication systems of pain, little is known about these relational effects. Previous findings indicate that children facially display pain to different degrees depending on the social partner in 'interaction' or 'transaction'. Thus, they show a higher degree of facial expressiveness in the presence of their parents, whereas they suppress their communication of pain in the presence of a stranger $(2-4)$. The few studies involving adults reveal similar results, with participants suppressing their facial responses during pain when an unfamiliar observer is present $(5,6)$. However, systematic investigations involving adults regarding the effects of no observer, 'strange' or 'familiar' observers on facial expressiveness remain lacking. In this

\section{L'influence des relations de communication sur les réponses faciales à la douleur : l'observateur a-t-il une importance?}

HISTORIQUE : On pense que les réponses faciales à la douleur sont un acte de communication. À ce titre, la relation entre l'émetteur et le récepteur est susceptible de les influencer.

OBJECTIFS : Examiner les conséquences des variations des relations de communication (être seul ou avec un proche) sur les éléments du langage facial utilisé pour communiquer la douleur (types de réponses faciales) et sur le degré d'expression faciale.

MÉTHODOLOGIE : Les chercheurs ont évalué les réponses faciales de 126 participants en santé à une douleur phasique causée par la chaleur dans trois situations sociales différentes : seul, mais conscient d'un enregistrement vidéo en cours, en présence d'un expérimentateur et en présence d'un proche. De plus, la catastrophisation de la douleur et le sexe (du participant et de l'expérimentateur) étaient considérés comme des influences supplémentaires.

RÉSULTATS : Les chercheurs ont observé des types de réponses faciales similaires quelle que soit la relation entre l'émetteur et l'observateur, mais le degré d'expression faciale variait considérablement et atteignaient un paroxysme en présence du conjoint. Fait intéressant, la présence d'un expérimentateur réduisait l'expression faciale seulement chez les femmes. La catastrophisation de la douleur et le sexe de l'expérimentateur n'avaient aucune influence importante sur les réponses faciales.

CONCLUSION : Les variations des relations de communication n'ont pas d'effet sur les éléments du langage facial de la douleur. Le degré d'expression faciale est toutefois adapté à la relation entre l'émetteur et le récepteur. Les individus suppriment leur communication faciale de la douleur devant des personnes non familières, mais l'expriment ouvertement en présence d'un proche. De plus, devant une personne non familière, diverses exigences situationnelles semblent entrer en jeu chez les deux sexes.

context, certain characteristics of the partners in 'interaction' or 'transaction' may constitute critical influences. A variable that may be of influence and should be considered is the sex of the observer. This assumption is based on previous findings showing that another form of pain communication, namely subjective verbal pain report, can vary according to the sex of the recipient (7-9), with men reporting less pain if the observer is female.

As suggested earlier, pain communication can be seen as 'action', which refers to the individual propensity or drive to display pain. Such individual factors are likely to preform or modulate, in turn, the 'interaction' and 'transaction'. To date, empirical investigations on the effects of these individual factors have been scarce and have produced sometimes inconsistent results. Pain catastrophizing is - although not undisputedly - assumed to be associated with high pain expressiveness (1) and may, therefore, affect communicative 'action' and, in turn, both 'interaction' and 'transaction'. In accord, only children scoring low on pain catastrophizing appear to adapt the degree of facial expressiveness to the social setting, while high pain-catastrophizing children's expressiveness remains constant across situations $(2,3)$. Another individual factor that likely influences the 'action' of pain 
communication and, thus, the 'interaction' and the 'transaction', is the sex of the individual displaying pain. This assumption can be derived from research investigating the facial display of other affective states, in which a modulating role of sex has been shown. Women (compared with men) facially display happiness and smiling to a higher degree $(10,11)$, whereas they suppress the facial expression of anger and aggression (12-15). Interestingly, this enhancement and suppression, respectively, also depends on the social setting (and thus on 'interaction' and 'transaction') (10-15). Considering these results, it is conceivable that the degree of facial expressiveness during pain is also different in high and low pain catastrophizers, and in men and women. More importantly, these individual factors of the sender may again be differentially affected by social relationships.

When reviewing previous research investigating the impact of social relationships on facial responses to pain, it is clear that all studies have concentrated on the degree of facial expressiveness, which solely refers to the vigor and number of facial responses shown (16). However, facial responses to pain are not only characterized by the degree of expressiveness but also by the elements of the facial language used to communicate pain (types of facial responses). It is known that pain is signalled by a limited number of facial actions, which occur across different modalities of experimental pain (eg, cold, electrical current), as well as across experimental and clinical pain conditions in a seemingly uniform fashion $(17,18)$. Due to this presumed consistency, the types of facial responses would not be expected to vary significantly depending on the recipient. However, even small variations within the limited number of facial actions may acheive communicative relevance. In the presence of an intimate other, an individual may, for example, favour signalling pain unpleasantness by eyebrow and levator contraction, whereas when alone, the same individual may selectively express the sensory dimension of his/her pain by contracting the muscles around the eyes (19). Thus, it appears reasonable to also determine whether the types of facial responses vary according to social relationships.

The aim of the present study was to further investigate how pain communication, considered as 'action', 'interaction' and 'transaction', is subject to changes in the relationship between sender and receiver. More precisely, we aimed to assess whether the types of facial responses occurring during painful stimulation and the degree of facial expressiveness vary among the following communicative relations: being alone (no observer, but aware of video recording); being with an experimenter (unfamiliar observer); and being with a partner (familiar observer). We hypothesized that the types of facial responses to pain would remain unaltered across the different situations (no qualitative changes of the facial pain language). On the other hand, we expected the degree of facial expressiveness during pain to be adapted to the relationship between participant and observer (only quantitative changes of the facial pain language). Given previous findings discussed above, we expected facial expressiveness to be reduced in the presence of the experimenter and to be elevated in the presence of an intimate other (in our case the partner). Furthermore, we considered pain catastrophizing and sex (of the participant and of the experimenter) to be additional critical influences on the effect that the relationship between sender and receiver has on the facial communication of pain.

\section{METHODS}

\section{Participants}

A total of 126 individuals (63 heterosexual couples; mean [ \pm SD] age $39.9 \pm 13.5$ years) participated in the current study. The participants were recruited via advertisements in the local newspaper (Bamberg, Germany). The advertisement recruited couples who had been in a relationship for $>6$ months $(20,21)$. Exclusion criteria were current experience of acute or chronic pain, psychological or physical illnesses, and paresthesia or other types of somatosensory dysfunctions affecting the left lower leg (site of stimulation). Participants taking psychotropic drugs or analgesics were also excluded from participation. All participants provided informed consent and received monetary compensation. The study protocol was approved by the ethics committee of the University of Bamberg (Bamberg, Germany).

\section{Procedure}

The experiment consisted of three sessions using thermal stimulation of painful and nonpainful intensities. In one session, participants received thermal stimulation while they were alone in the room. During the other two sessions, another individual was present during the testing. This person was their partner in one session and an experimenter (male or female; randomly assigned) in another session. Thus, each subject was a participant in one session and an observer in another. Participants were informed that the focus of interest was how pain responses change across time and across social situations. Before testing started, the experimenter gave instructions, explained the following procedure and ensured everything was understood. The participants were asked to complete the German version of the Pain Catastrophizing Scale (PCS) at the end of the experiment, to avoid directing the participants' focus on pain-related thoughts before the painful stimulation.

\section{Social manipulation}

In the 'alone' session, the experimenter left the room immediately before thermal stimulation started. Participants were informed that the experimenter would be in the adjoining room and would return at the end of the stimulation session, or if they vocally signalled the need for anything. The participants were aware of being videorecorded (the video recording was kept constant across all three situations and, thus, participants were aware of video recording during all three sessions).

In the session with an experimenter present, the experimenter remained in the room and was seated in front of the computer controlling the thermal stimulation, which was located slightly on the right of the participant, approximately $2 \mathrm{~m}$ away. Participant and experimenter were facing one another and were able to have eye contact. However, the experimenter was instructed to avoid any verbal communication.

In the session with the partner present, the participant was in the room with his or her partner, seated slightly to the left, approximately $2 \mathrm{~m}$ away (without the experimenter being present). Similar to the session with an experimenter present, the participant and partner were facing one another and able to have eye contact, but were instructed not to talk to one another during stimulation. The seating positions of partner and experimenter resulted from the experimental setting, in which the experimenter sat in front of the computer controlling the thermal stimulation whereas the partner was seated away from the stimulator.

The order of the sessions was randomized across participants. The experiment always began with one partner undergoing all three sessions of thermal stimulation and then continued with the other partner as the participant. To avoid order effects, the female participant was tested first in $50 \%$ of the couples; in the other $50 \%$, male participants were tested first.

\section{Stimulation}

Thermal stimulation was applied to three designated sites on the outer part of the left lower leg by a Peltier-based contact stimulation device (TSA-2001, Medoc, Israel) with a $30 \mathrm{~mm} \times 30 \mathrm{~mm}$ contact thermode. The lower leg was chosen as the site for stimulation because it provides sufficient space to alter thermode placement between sessions and provided a rest period of at least $15 \mathrm{~s}$ between single stimuli; these two measures should prevent sensitization.

To ensure that temperature intensities were perceived as painful but not too painful in all participants (to prevent floor as well as ceiling effects), temperature intensities were tailored to the individual pain threshold. Thus, heat pain thresholds were determined first, using the method of adjustment. Participants were asked to adjust a temperature starting from $38^{\circ} \mathrm{C}$, using heating and cooling buttons, until they obtained a level which was barely painful. A constant press of the buttons produced a heating or cooling rate of $0.5^{\circ} \mathrm{C} / \mathrm{s}$. Following a 
familiarization trial, there were four trials and the average of these trials was used to constitute the threshold estimate.

Following the assessment of pain thresholds, phasic heat stimuli ( $5 \mathrm{~s}$ [plateau]; rate of change: $4^{\circ} \mathrm{C} / \mathrm{s}$; baseline temperature: $38^{\circ} \mathrm{C}$; interstimulus intervals of $15 \mathrm{~s}$ to $20 \mathrm{~s}$ ) were applied to the lower leg. Two different stimulus intensities were applied; namely, painful $\left(+3^{\circ} \mathrm{C}\right.$ above the pain threshold) and nonpainful $\left(-3^{\circ} \mathrm{C}\right.$ below the pain threshold) intensities. Applying nonpainful intensities allowed for the determination of which types of facial responses are specific for painful experiences. In each experimental session, participants received 10 painful and 10 nonpainful stimuli in random order.

\section{Dependent variables}

Self-report ratings: Participants were asked to provide self-report ratings using an electronic visual analogue scale (VAS; $100 \mathrm{~mm}$ ), which appeared horizontally on a computer screen after each phasic stimulus. The scale was labelled with a verbal anchor of 'faintly painful' in the centre; thus, all nonpainful sensations should be rated below and all painful ones above. Participants were informed that the left and right ends of the scale corresponded to 'no sensation' and 'extremely strong pain', respectively. Participants were asked to rate the intensity of their nonpainful and painful experiences by moving the mouse cursor to the right or left, thereby choosing one location on the scale. This cursor appeared in a random location on the scale each time it was presented to avoid biases due to one-sided starting positions. Ratings had to be given within $10 \mathrm{~s}$ after stimulus offset.

Facial expression: Participant's faces were videotaped throughout the pain induction procedures. The camera was located approximately $1.0 \mathrm{~m}$ from the participant on top of the computer screen and participants were informed of the video recording. To enable offline segmentation of the videos, an LED light visible to the camera, but not to the participant, was lit concurrently with the $5 \mathrm{~s}$ thermal stimulation, beginning when the target temperature was reached. To ensure that the face would always be upright and in a frontal view during stimulation, participants were asked to look at the computer screen in front of them throughout the whole session and rate stimuli after they had appeared. Participants were also instructed not to talk during thermal stimulation

Facial expressions were coded from the video recordings using the Facial Action Coding System (FACS) (22), which is based on anatomical analysis of facial movements and distinguishes 44 different 'action units' (AUs) produced by single muscles or combinations of muscles. Three coders, trained by a certified FACS coder (qualified by passing an examination given by the developers of the system) identified the frequency and the intensity (five-point scale) of the different AUs (inter-rater reliability, calculated using the Ekman-Friesen formula [22], was between 0.84 and 0.87). Software designed for the analysis of observational data (Observer Video-Pro; Noldus Information Technology, Netherlands) was used to segment the videos and to enter the FACS codes into a time-related database. Time segments of $5 \mathrm{~s}$ beginning just after the stimulus had reached the target temperature (time period during which the LED was lit) were selected for scoring. In total, $3 \times 20$ segments of thermal stimulation (10 nonpainful and 10 painful segments in three sessions) were analyzed for each participant. For the purpose of necessary data reduction, AUs that represent facial movements of the same muscle were combined, as has been performed in previous studies without any loss of information $(23,24)$. Those combinations include AU 1/2,6/7, 9/10 and 25/26/27.

\section{Independent variables}

Pain catastrophizing: A German translation of the PCS was used to assess catastrophic thinking related to pain (25). Participants were instructed to reflect on thoughts or feelings during the past painful experiences. The scale contains 13 items that are rated on a five-point scale, with the end points 'not at all' and 'all the time'. The PCS has been widely used in research on pain catastrophizing, and has been shown to have high internal consistency (Cronbach's $\alpha=0.87)(25,26)$. Sex: In addition to the PCS score, sex of the experimenter and participant were considered and used as independent variables in the following analyses.

\section{Statistical analyses}

Because participants were recruited as couples, the population investigated in the current study cannot be considered to be a perfect random sample. Thus, as well as being a participant who experienced pain, each participant was also once the observer. To exclude that the degree of facial expressiveness of those participants who first served as an observer was influenced by observing their partners' degree of facial expressiveness, the association between the partners' degree of facial expressiveness was assessed. This was performed by correlating the degree of facial expressiveness (composite score; see 'Degree of facial expressiveness' below) between partners separately for all three situations. None of these correlations were significant (all $\mathrm{P}>0.05$ ). In addition, when correlating the single $\mathrm{AUs}$ ( $\mathrm{AU} 4,6 / 7,9 / 10$ ) separately, which were later aggregated into the composite score, none of the correlations between partners were significant (all $\mathrm{P}>0.05$ ). This was the case for all three situations. Therefore, a lack of independence of the data cannot be assumed, which, in the following analyses, allows the participants to be treated as individuals who had been recruited by conventional sample methods.

\section{Effect of communicative relations on pain responses}

Self-report: The impact of communicative relations (alone, in the presence of the experimenter or in the presence of the partner) on subjective pain ratings was investigated using repeated-measures ANOVA (dependent variable: VAS ratings; within-subject factor: communicative relation)

Facial expression: Types of facial responses: One aim of the present study was to assess whether different types of facial responses occur during painful stimulation depending on the communicative relation. This was assessed by first determining which AUs were displayed in each of the three social situations at a frequency $>5 \%$ (of the painful segments). The critical margin of $5 \%$ was derived from earlier studies (23,27-31). Subsequently, whether the frequency distribution of these AUs was comparable in all three social situations (alone, with partner and with experimenter) was analyzed. To test for differences in frequency distribution of AUs (percentage of occurrence of each AU) among social situations, $\chi^{2}$ analyses were conducted comparing distributions between alone versus experimenter, alone versus partner and experimenter versus partner.

Moreover, which of the AUs with an occurrence frequency $>5 \%$ were truly pain indicative and whether different AUs may be pain indicative in different communicative relations was determined. To determine this, effect sizes (Cohen's d for repeated measures) contrasting occurrence frequencies of each AU between painful and nonpainful trials were computed. This was calculated separately for each social situation. AUs showing an effect size of $\mathrm{d} \geq 0.5$ (medium effect) were selected as pain-indicative facial responses, and whether the same AUs proved to be pain indicative in all three social situations was descriptively compared.

Degree of facial expressiveness: In addition to comparing the types of facial responses to pain between social situations, whether the degree of facial expressiveness was affected by the type of relationship between the participant and observer was also investigated. To do this, all AUs that proved to be pain-indicative (see description above) across all three social situations were combined into one composite score of pain-relevant facial responses. The composite score was calculated by first combining the frequency and intensity values of each $\mathrm{AU}$ to product terms $(19,23)$. Second, the product terms of all painrelevant AUs were averaged. Finally, the averaged product terms were square-root transformed to yield unskewed composite scores of painrelevant facial responses, as has been performed in previous studies $(19,24)$. Composite scores were calculated for each of the three situations separately. Therefore, it was possible to conduct a repeatedmeasures ANOVA to assess the effect of communicative relations (alone, with partner and with experimenter) on the composite scores of pain-relevant AUs (degree of facial expressiveness). 


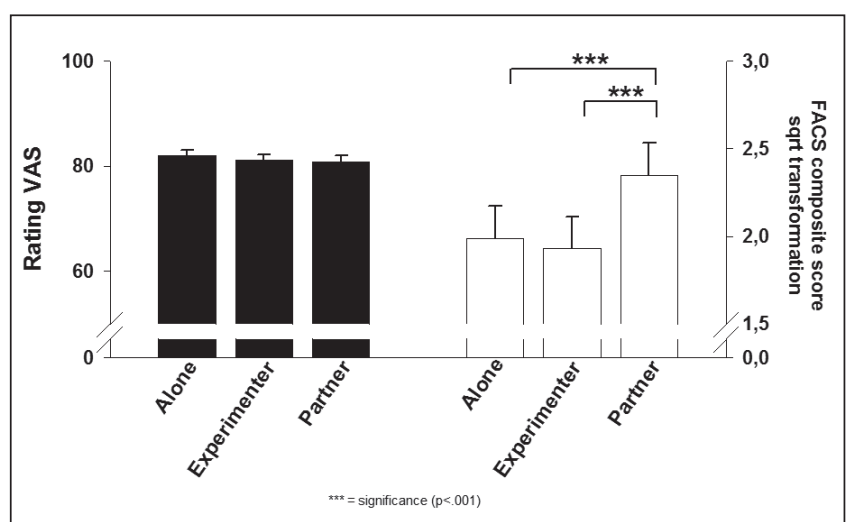

Figure 1) Mean \pm SEM values for visual analogue scale (VAS) ratings of subjective pain intensity (left); and composite scores (square-root [sqrt] transformed) of evoked (pain-relevant) facial expressiveness in response to painful heat stimulation (right). Values are given separately for all three social situations (alone, experimenter and partner). FACS Facial Action Coding System

\section{Additional influences}

To determine whether the effect of communicative relations on the degree of facial expressiveness was also affected by other factors (ie, pain catastrophizing, sex of the participant and sex of the experimenter), additional analyses were necessary. To determine how those factors affected the changes in the degree of facial expressiveness, change scores of facial expressiveness between social situations were calculated to serve as dependent variables. This approach was also necessary due to the study design. Because the sex of the experimenter was only present as a variable in the experimenter session and, thus, could not have affected facial expressiveness when participants were alone or with their partner, including all three factors in one comprehensive analysis was not possible. Therefore, change scores of facial expressiveness between the social situations were calculated with the 'alone' condition serving as baseline and fed into two distinct analyses, investigating:

- the change in expressiveness from being alone to being with the experimenter: An ANOVA was performed to determine whether the change in expressiveness from being alone to being with the experimenter (difference score: experimenter - alone) was influenced by the sex of the participant and by the sex of the experimenter, as well as by pain catastrophizing (median split of PCS score). All three variables were entered as independent variables into the ANOVA; and

- the change in expressiveness from being alone to being with their partner: This second ANOVA examined whether variations in expressiveness from being alone to being with their partner (difference score: partner - alone) were influenced by the sex of the participant (as previously mentioned, the sex of the observer was of no interest in this context because only heterosexual couples were evaluated and, thus, the sex of the observer was always opposite to the sex of the participant) and by pain catastrophizing (median split of PCS score). Both variables were entered as independent variables into the ANOVA.

Because these ANOVAs used change scores, whether PCS, sex of the participant and sex of the experimenter affected the degree of facial expressiveness in general could not be determined. Therefore, $t$ tests assessing the general influence of sex and PCS on overall facial expressiveness (composite score merged over all three situations) were also computed.

Results for the within-factor analyses were corrected according to Greenhouse-Geisser whenever the Mauchly test of sphericity indicated heterogeneity of covariance. For ANOVAs showing significance, post hoc $t$ tests were calculated. Findings were considered to be statistically significant at $\alpha<0.05$. In addition to P values, partial eta-squared $\left(\eta_{\mathrm{p}}{ }^{2}\right)$ and Cohen's $\mathrm{d}$ as measures of effect size were also reported.
TABLE 1

Facial Action Units (AUs) with a critical frequency of occurrence $>5 \%$ in painful segments. Data are presented for each social situation

\begin{tabular}{|c|c|c|c|c|c|c|}
\hline \multirow[b]{2}{*}{$A U$} & \multicolumn{2}{|c|}{ Alone } & \multicolumn{2}{|c|}{ Partner } & \multicolumn{2}{|c|}{ Experimenter } \\
\hline & Percent ${ }^{*}$ & $\begin{array}{c}\text { Effect } \\
\text { size }\end{array}$ & Percent ${ }^{*}$ & $\begin{array}{c}\text { Effect } \\
\text { size }\end{array}$ & Percent ${ }^{*}$ & $\begin{array}{c}\text { Effect } \\
\text { size }\end{array}$ \\
\hline $\mathrm{AU} 1 / 2$ & 14.1 & 0.2 & 18.6 & 0.3 & 12.0 & 0.3 \\
\hline$A U 4$ & 29.2 & 0.7 & 35.2 & 0.9 & 29.8 & 0.7 \\
\hline AU6/7 & 44.5 & 0.6 & 55.2 & 0.7 & 44.0 & 0.7 \\
\hline AU $9 / 10$ & 24.0 & 0.5 & 25.3 & 0.5 & 19.8 & 0.6 \\
\hline AU 12 & 7.8 & 0.4 & 13.0 & 0.2 & - & - \\
\hline AU 14 & 11.4 & 0.1 & 10.5 & 0.0 & 14.1 & 0.3 \\
\hline AU 17 & 5.0 & 0.1 & 8.1 & 0.2 & 6.9 & 0.2 \\
\hline AU 18 & 8.2 & 0.5 & 7.1 & 0.5 & 6.1 & 0.3 \\
\hline AU 23 & 5.8 & 0.2 & 5.6 & 0.3 & 6.0 & 0.3 \\
\hline$A \cup 25 / 26$ & 35.3 & 0.6 & 29.6 & 0.4 & 27.2 & 0.3 \\
\hline
\end{tabular}

Effect sizes for frequency differences between nonpainful and painful segments are given. Medium and strong effect sizes $(d \geq 0.5)$ are marked in bold. ${ }^{*}$ Denotes the percentage of occurrence in the entire painful segments

\section{RESULTS}

In the present study, 63 heterosexual couples $(n=126)$ were tested. The mean age of the participants was $39.9 \pm 13.5$ years and the mean length of the relationship was $14.8 \pm 13.3$ years; mean pain threshold was $46.3 \pm 1.2^{\circ} \mathrm{C}$. Given the healthy and pain-free sample of individuals tested, it is not surprising that the median score of the PCS was fairly low (14.0). Accordingly, the subjects were separated into two groups based on the median split: 'low' (PCS score range 0 to 14 ) and 'moderate' (PCS score range 15 to 36 ) pain catastrophizers. The two groups did not differ significantly with regard to age and pain threshold (all $\mathrm{P}>0.6$ ). In addition, there were almost equal numbers of male and female participants in each group (low PCS group, $49.3 \%$ female participants; moderate PCS group, 52.5\% female participants).

Effect of communicative relations on pain responses

Self-report: Self-report ratings, as provided by the VAS, did not change across social situations $(F[2,250]=1.528, P=0.219, \eta=0.012)$ (Figure 1). On average, participants rated painful stimulation as $82.0 \pm 11.7$ when being alone, $81.1 \pm 13.1$ when in the presence of the experimenter and $80.8 \pm 12.8$ when with their partner (on a scale ranging from 0 to 100 , with 50 representing 'faintly painful').

Facial expression: Types of facial responses to pain: There were only very minor frequency differences among the three social situations with regard to the types of facial responses to pain occurring with a frequency of $>5 \%$ (Table 1). With the exception of AU 12 (which did not occur above the critical level of $>5 \%$ in the experimenter situation), the same AUs were displayed independently of the communicative relation. Moreover, when comparing the frequency distribution of those AUs between situations ( $\chi^{2}$ analyses of frequencies), no differences were observed. The distribution of AUs when being alone did not significantly differ from the AU distribution in the presence of the partner $\left(\chi^{2}=1.59 ; \mathrm{P}=0.991\right)$ or in the presence of an experimenter $\left(\chi^{2}=1.207\right.$; $\mathrm{P}=0.997)$. The distribution of AUs did also not significantly differ between the 'partner' and 'experimenter' session $\left(\chi^{2}=1.262 ; \mathrm{P}=0.996\right)$. Brow lowering (AU 4), orbit tightening (AU 6/7) and levator contraction $(\mathrm{AU}$ 9/10) were the facial responses displayed most frequently regardless of the social situation. Moreover, when considering which of the AUs proved to be indicative of pain in each of the three situations (effect sizes $\geq 0.5$ for the difference in frequency between nonpainful and painful stimulation), high agreement was again observed among the social situations (Table 1). Therefore, the type of relationship between the participant (sender) and observer does not appear to have a strong effect on the types of facial responses being elicited during pain. 


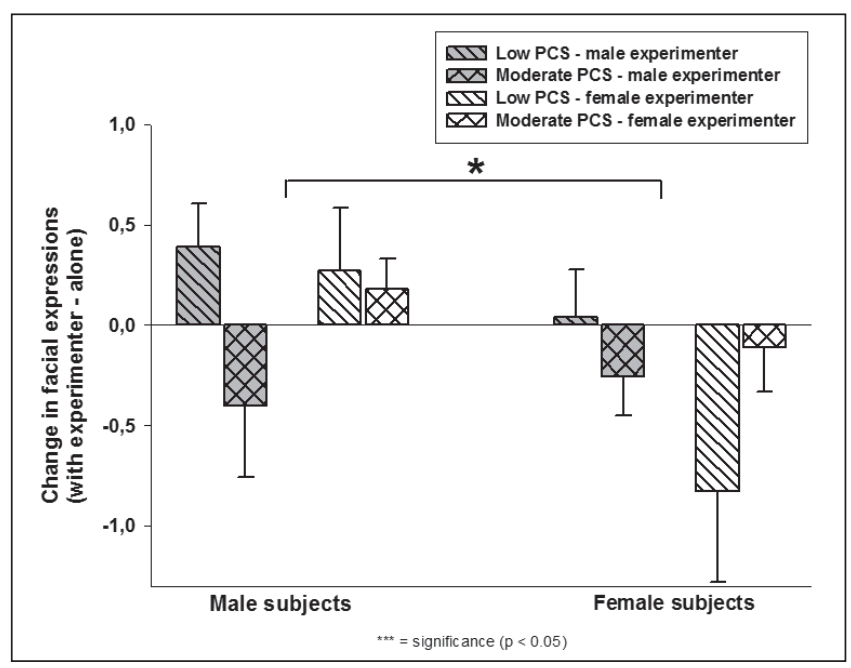

Figure 2) Change scores of facial expressiveness (difference of the composite scores of facial expressiveness (square-root transformed) in the situations 'experimenter - alone'; mean \pm SEM values. Scores are presented separately for male and female participants as well as experimenters, and for low and moderate pain catastrophizers. PCS Pain Catastrophizing Scale

Degree of facial expressiveness: When investigating whether the communicative relation affects the degree of facial expressiveness, however, major differences were apparent. The composite score consisting of the AUs that were indicative of pain in all three situations (brow lowering [AU 4], orbit tightening [AU 6/7] and levator contraction [AU9/10]) differed substantially among the situations ( $F[2,250]=9.771$, $\mathrm{P}<0.001, \eta=0.072$ ) (Figure 1). Post hoc testing showed that, in the presence of the partner, participants significantly increased the degree of facial expressiveness (elevated composite score) compared with being alone $(t[126]=-3.64, \mathrm{P}<0.001)$ or in the presence of the experimenter $(t[126]=-3.82, \mathrm{P}<0.001)$. When comparing the situation of being alone with the presence of the experimenter, no significant differences were observed $(t[126]=0.56, P=0.574)$.

\section{Additional influences}

Alone versus experimenter: Results of the additional ANOVA analyzing the influence of participant sex and experimenter sex, as well as the participant's PCS level on the change of facial expressiveness due to the presence of an experimenter compared with being alone, are presented in Figure 2.

The only main effect that reached significance was the sex of the participant $(F[1,124]=4.025, P=0.047, \eta=0.034)$. As shown in Figure 2, the change score of female participants was almost consistently negative, while that of male participants varied but tended to be positive. Thus, female participants reduced their facial expressiveness during painful stimulation in the presence of an experimenter compared with being alone, while male participants showed no consistent change in expressiveness (Figure 3). By itself, neither the sex of the experimenter ( $F[1$, $124]=0.112, \mathrm{P}=0.739)$ nor the PCS score $(\mathrm{F}[1,124]=0.345, \mathrm{P}=0.558)$ had a significant influence. The two-way interaction between those factors, however, reached significance $(F[1,124]=4.635, P=0.033, \eta=0.038)$. Pairwise comparisons showed that this effect was solely caused by the significant differences between moderate and low pain catastrophizers in the presence of a male experimenter $(t[65]=-2.056, \mathrm{P}=0.044)$. Compared with low pain catastrophizers, moderate pain catastrophizers decreased their facial expressiveness when a male experimenter was present. Aside from this interaction, none were significant (all $\mathrm{P}>0.05$ ).

Alone versus partner: Results of the additional ANOVA concerning the influence of the sex of the participant and the participant's PCS level on the change of facial expressiveness due to the presence of the

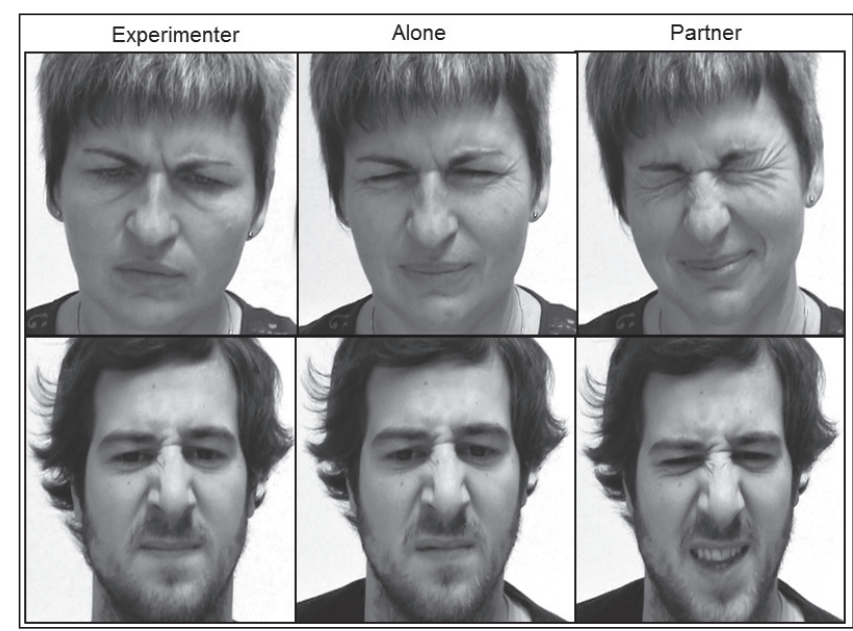

Figure 3) Examples of evoked facial expressions during painful stimulation in each of the three social situations by a female (upper row) and male participant (lower row)

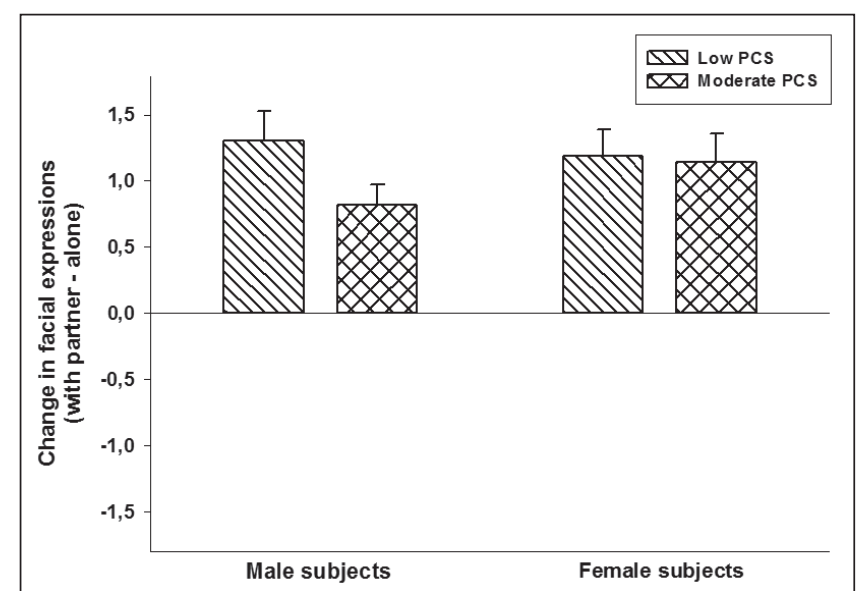

Figure 4) Change scores of facial expressiveness (difference of the composite scores of facial expressiveness (square-root transformed) in the situations 'partner-alone'; Mean values ( \pm SEM). Scores are presented separately for male and female participants and for low and moderate pain catastrophizers

partner compared with being alone are presented in Figure 4. Given that the experimenter was neither present in the 'alone' nor in the 'partner' situation, the sex of the experimenter was not considered in this analysis.

Neither the PCS score $(F[1,124]=0.158 ; \mathrm{P}=0.692)$ nor the sex of the participant $(F[1,124]=0.712 ; \mathrm{P}=0.401)$ had an impact on the change in facial expressiveness in the presence of a partner compared with being alone. Furthermore, there was no significant interaction between these factors (all $\mathrm{P}>0.05$ ).

General effects of individual participant factors (sex and PCS)

The general effects of participant sex and pain catastrophizing on the overall degree of facial expressiveness were also evaluated independent of the social situations. $t$ tests for independent samples showed that neither PCS nor sex of the participant had a significant general impact on facial expressiveness during painful stimulation (all $\mathrm{P}>0.15)$.

\section{DISCUSSION}

The present study was designed to investigate whether, and how, different communicative relations (alone [but aware of video recording], with the partner, with an experimenter) affect facial responses to pain 
in healthy adults. Our results indicate that pain is signalled by the same types of facial responses independent of the type of relationship between participant (sender) and observer. However, the degree of facial expressiveness, which represents the strength of the social signal, varied and was apparently adjusted according to social rules. In other words, facial responses to pain appeared to be similar when participants were alone, with a stranger or with an intimate partner; however, the intensity of the response varied dependently on the recipient. These findings will be discussed in detail below.

\section{Effect of communicative relations}

The effect of communicative relations on facial responses to pain was investigated by considering the types of facial responses and the degree of facial expressiveness.

With regard to the types of facial responses, we found no significant differences among the three situations. As expected, similar types of facial responses to pain were elicited with similar percentage distributions regardless of the type of relationship between participant (sender) and observer. These responses included the lowering of the brow (AU 4), orbit tightening (AU 6/7) and levator contraction (AU 9/10), all of which have been previously reported in the context of pain $(16,17,32)$. Given that the facial response to pain has to be recognized with high certainty - to fulfill its 'interactional' and 'transactional' goals $(1,33)$ - finding it to be unchanged across social situations seems reasonable. If the communicative relation fundamentally changes the types of facial responses being elicited during pain, the certainty of recognition in 'interaction' and 'transaction' may be endangered. Therefore, displaying the same types of responses independently of the relationship between sender and observer most likely preserves the essential recognisability of the facial expression of pain.

However, we found the degree of facial expressivenessto be significantly affected by communicative relations. Whereas the degree of facial expressiveness was significantly elevated when an intimate other, in this case the partner, was present, the presence of the experimenter caused a tendency to reduce facial expressiveness (only in women). Accordingly, individuals appear to adjust the vigor and number of their facial responses depending on the relationship between sender and observer and, thus, depending on the different 'interactional' and 'transactional' goals.

A similar effect has been shown for other affective states (eg, anger, joy) and has been interpreted in terms of so-called 'social display rules'. These display rules define whether and how an expression of emotion is culturally appropriate in a given social situation $(13,15,34,35)$. For example, in the presence of a formal other (eg, teacher, experimenter) it seems less appropriate to overtly show one's emotions $(35,36)$, whereas emotions can be communicated more openly toward intimate others (eg, parent or friend) $(35,37)$. Our results, along with previous findings in children $(2,3)$, indicate that the facial expression of pain follows similar situational standards. When being in the presence of an intimate other - the partner (our study) or a parent $(2,3)$ - individuals appear to communicate their pain more strongly via facial expressions compared with being alone or in the presence of a stranger. Why might it be an advantage to adapt one's facial expressiveness during pain dependent on the recipient?

Given that facial responses to pain can be a powerful means of eliciting empathy and social support in the observer $(33,38)$, it is reasonable to communicate pain to a higher extent toward observers who are sympathetic, because they are the ones who would most likely provide help. Consistent with this, it has been shown that the amount of time that two individuals commonly spend with one another predicts whether these individuals attend to and correctly interpret one another's pain behaviour (39). Therefore, it seems sensible for an individual to display pain more openly toward those observers whom they spend a lot of time with - such as the partner - and who may be more willing to attend to and more able to decode the sent message. On the other hand, it has been hypothesized that showing vulnerability by signalling pain to observers whose reactions one cannot predict (an unfamiliar other) may endanger the individual who is experiencing pain $(33,40,41)$.

When interpreting the current data, it has to be noted that participants were aware of video recording during all aspects of the experiment and, thus, participants may have felt 'observed' in all three conditions. However, this awareness of being observed by a camera may have interfered in particular with the experimental goal in the 'alone' situation, in which we aimed to assess the individual when he/ she believed they were alone or, in other words, unobserved. Therefore, the degree of facial expressiveness in our 'alone' situation may have been lower than when participants were truly convinced they were alone and completely unobserved. This, in turn, may have contributed to the lack of differences when comparing the expressiveness in the presence of an unfamiliar other (the 'experimenter' situation) with the 'alone' situation.

Additional influences on the effect of communicative relations Alone versus experimenter: The change in the degree of expressiveness due to the presence of the experimenter was significantly influenced by the sex of the participant. Women - in contrast to men - significantly decreased the degree of facial expressiveness during pain in the presence of an experimenter compared with being alone. This finding is consistent with previous findings on sex differences in social display rules. In front of an unfamiliar other, females facially express positive affective states, such as happiness, to a higher degree, whereas they conceal negative ones such as anger $(11-13,15,35)$. Given that pain is a rather negative affective state, the decrease of womens' facial expressiveness in the presence of an experimenter is consistent with these previous results.

As opposed to the sex of the participant, there was no main effect found for the sex of the experimenter. Participants showed comparable levels of facial expressiveness, regardless of whether the experimenter was male or female. This result is inconsistent with previous findings on subjective pain reports, which have shown that male research participants rate stimuli as less painful when being tested by a female compared with a male experimenter (7-9). It is possible that facial and subjective pain responses are differentially affected by the sex of the experimenter. Moreover, previous studies more strongly emphasized gender roles (by specific clothing and behaviour) than we did (42) and, thus, we may have neutralized gender roles.

Earlier studies found pain catastrophizing to moderate the effect that familiar or unfamiliar observers may have on facial expressiveness $(2,3,6)$. However, similar to a previous study of ours (23), pain catastrophizing neither had an effect on the overall degree of facial expressiveness nor on the effect of familiarity of observer (communicative relations) on this parameter in the present study. The reason for this inconsistency may originate in methodical differences such as the type of participants (children versus adults), type of pain stimulation (fixed physical versus psychophysically adjusted stimulus intensities) and type of facial analysis (Children Facial Coding System (43) versus FACS). We found a significant interaction only between catastrophizing and sex of the experimenter. Rather puzzlingly, moderate pain catastrophizers, compared with low catastrophizers, reduced the degree of facial expressiveness in the presence of a male experimenter. Therefore, further research seems necessary to specify whether and how pain catastrophizing plays a role in modulating the effects of communicative relations on facial responses to pain.

Alone versus partner: The shift to stronger facial expressiveness in the presence of a partner was neither influenced by pain catastrophizing nor by the sex of the participant. We speculate that the drive to signal pain to the partner is so pronounced that variables, such as sex and pain catastrophizing, cannot add to that and, thus, become less relevant.

\section{Self report}

In addition, we assessed self-report ratings to control whether differences in facial responses between situations are simply caused by 
differences in subjective experiences. To minimize the impact of communicative relations on self-report ratings, a computerized assessment was performed, with research participants always rating the stimuli on a computer screen that was neither visible to the partner nor to the experimenter. Given that self-report ratings did not change between social situations, we can exclude the possibility that the observed changes in facial expressiveness are only due to changes in subjective pain experience.

\section{Limitations}

As mentioned above, one limitation to the present study is the fact that participants also knew that they were being filmed when being alone. In future replications, it would be interesting to also include a condition in which participants genuinely believe themselves to be alone; however, due to ethics constraints, this would be difficult to perform.

Another limitation to the current study was the arrangement of seating positions in the experimental room. The experimenter always sat contralateral whereas the partner always sat ipsilateral to the stimulation site. As has been shown (44), the resulting different eye orientations during stimulation may have affected our results. However, finding no differences in self-report ratings between the situations appears to contradict this assumption.

In addition, the special feature of having tested couples has been underused in the current study because, for the purposes of the present study, this form of recruitment only guaranteed the familiarity of the partner but was not considered for other aspects of partnership. We may be able to deliver these data at a later point.

As a final point of potential criticism, we grouped our subjects into 'low' and 'moderate' PCS scorers by using the median as cut-off, which is a somewhat arbitrary criterion. Furthermore, this approach, which we preferred for its good illustrative properties, reduced individual differences. However, even when using raw PCS scores in a regression design the same results evolve, with PCS scores neither being able to predict significantly the change of facial expressiveness between being 'alone' and 'with the experimenter' nor the change of facial expressiveness between being 'alone' and 'with the partner' (all P>0.05). Therefore, we can exclude the possibility of crucial information loss due to using the median-split approach.

\section{CONCLUSION}

Independently of the recipient, pain is signalled via the same types of facial responses. Thus, the elements of the facial language used to communicate pain remain unaltered by the relationship between sender and observer, which may guarantee reliable recognition. However, the degree of facial expressiveness changes depending on the communicative relations, with increasing expressiveness occurring in the presence of an intimate other. This appears to be beneficial because sympathetic observers may be able to more quickly identify painful experiences and, therefore, the possibility of receiving help is elevated. Interestingly, when confronted with an unfamiliar individual, the two sexes appear to pursue different 'interactional' or 'transactional' goals. Whereas men do not alter the degree of facial expressiveness when being observed by an experimenter (compared with being alone), women reduce their degree of facial expressiveness, possibly due to learned display rules that make the overt display of negative affect in women seem inappropriate.

ACKNOWLEDGEMENTS: The authors thank Viktoria Zorn and Nina Kunzelmann for their support in conducting the study and the FACS coding.

DISCLOSURES: The study was supported by a research grant from the Deutsche Forschungsgemeinschaft (DFG, Ku2294/4). The authors have no financial or other relationships that may lead to a conflict of interest.

\section{REFERENCES}

1. Hadjistavropoulos T, Craig KD, Duck S, et al. A biopsychosocial fomulation of pain communication. Psychol Bull 2011;137:910-39.

2. Vervoort T, Caes L, Trost Z, Sullivan M, Vangronsveld K, Goubert L. Social modulation of facial pain display in highcatastrophizing children: An observational study in schoolchildren and their parents. Pain 2011;152:1591-9.

3. Vervoort T, Goubert L, Eccleston C, et al. The effects of parental presence upon the facial expression of pain: The moderating role of child pain catastrophizing. Pain 2008;138:277-85.

4. Zeman J, Garber J. Display rules for anger, sadness, and pain: It depends on who is watching. Child Dev 1996;67:957-73.

5. Kleck RE, Vaughan RC, Cartwright-Smith J, Vaughan KB, Colby C, Lanzetta J. Effects of being observed on expressive, subjective, and physiological reactions to painful stimuli. J Personality Soc Psychol 1976;34:121-8.

6. Vlaeyen JW, Hanssen M, Goubert L, et al. Threat of pain influences social context effects on verbal pain report and facial expression. Behav Res Ther 2009;47:774-82.

7. Aslaksen PM, Myrball IN, Hoifodt RS, Flaten MA. The effect of experimenter gender on autonomic and subjective responses to pain stimuli. Pain 2007;129:260-8.

8. Kállai I, Barke A, Voss U. The effects of experimenter characteristics on pain reports in women and men. Pain 2004;112:142-7.

9. Levine FM, De Simone LL. The effects of experimenter gender on pain report in male and female subjects. Pain 1991;44:69-72.

10. Hall JA. Nonverbal Sex Differences: Communication Accuracy and Expressive Style. Baltimore: The John Hopkins University Press, 1984.

11. LaFrance M, Hecht MA, Levy Paluck E. The contingent smile: A meta-analysis of sex differences in smiling. Psychol Bull 2003;129:305-34.

12. Cole P. Children's spontaneous control of facial expression. Child Dev 1986;57:1309-21.

13. Davis TL. Gender differences in masking negative emotions: Ability or motivation? Dev Psychol 1995;31:660-7.

14. Kring AM. Gender and anger. In: Fischer AH, ed. Gender and Emotion. Cambridge, United Kingdom: Cambridge University Press, 2002:211-31.

15. Saarni C. An observational study of children's attempts to monitor their expressive behavior. Child Dev 1984;55:1504-13

16. Craig KD, Prkachin KM, Grunau R. The facial expression of pain. In: Turk D, Melzack R, eds. Handbook of Pain Assessment. New York: Guilford Press, 2011:117-33.

17. Prkachin KM. The consistency of facial expressions of pain: A comparison across modalities. Pain 1992;51:297-306.

18. Prkachin KM, Solomon PE. The structure, reliability and validity of pain expression: Evidence from patients with shoulder pain. Pain 2008;139:267-74

19. Kunz M, Lautenbacher S, LeBlanc N, Rainville P. Are both the sensory and the affective dimensions of pain encoded in the face? Pain 2012;153:350-8.

20. Downey G, Freitas AL, Michaelis B, Khouri H. The self-fulfilling prophecy in close relationships: Rejection sensitivity and rejection by romantic partners. J Personal Soc Psychol 1998;75:545-60.

21. Grewen KM, Anderson BJ, Girdler SS, Light KC. Warm partner contact is related to lower cardiovascular reactivity. Behav Med 2003;29:123-30.

22. Ekman PE, Friesen WV. Facial Action Coding System. Palo Alto: Consulting Psychologists Press, 1978.

23. Kunz M, Chatelle C, Lautenbacher S, Rainville P. The relation between catastrophizing and facial responsiveness to pain. Pain 2008;140:127-34

24. Kunz M, Faltermeier N, Lautenbacher S. Impact of visual learning on facial expressions of physical distress: A study on voluntary and evoked expressions of pain in congenitally blind and sighted individuals. Biol Psychol 2012;89:467-76.

25. Sullivan MJ, Bishop SR, Pivik J. The pain catastrophizing scale: Development and validation. Psychol Assess 1995;7:524-32.

26. Sullivan MJ, Martel MO, Tripp D, Savard A, Crombez G. The relation between catastrophizing and the communication of pain experience. Pain 2006;122:282-8.

27. Hadjistavropoulos HD, Craig KD, Hadjistavropoulos T, Poole GD. Subjective judgments of deception in pain expression: Accuracy and errors. Pain 1996;65:251-8. 
28. Hadjistavropoulos T, LaChapelle DL, MacLeod FK, Snider B, Craig KD. Measuring movement-exacerbated pain in cognitively impaired frail elders. Clin J Pain 2000;16:54-63.

29. Kunz M, Mylius V, Schepelmann K, Lautenbacher S. Impact of age on the facial expression of pain. J Psychosom Res 2008;64:311-8.

30. Kunz M, Mylius V, Schepelmann K, Lautenbacher S. On the relationship between self-report and facial expression of pain. J Pain 2004;5:368-76.

31. Kunz M, Scharmann S, Hemmeter U, Schepelmann K, Lautenbacher $\mathrm{S}$. The facial expression of pain in patients with dementia. Pain 2007;133:221-8.

32. Prkachin KM, Craig KD. Expressing pain: The communication and interpretation of facial pain signals. J Nonverbal Behav 1995:19:191-205.

33. Williams AC. Facial expression of pain: An evolutionary account. Behav Brain Sci2002;25:439-55.

34. Brody LR. The socialization of gender differences in emotional expression: Display rules, infant temperament, and differentiation. In: Fischer AH, ed. Gender and Emotion. Cambridge: Cambridge University Press, 2002:24-47.

35. Underwood MK, Coie JD, Herbsman CR. Display rules for anger and aggression in school-age children. Child Dev 1992;63:366-80.

36. Yarczower M, Daruns L. Social inhibition of spontaneous facial expression in children. J Pers Soc Psychol 1982;43:831-7.
37. Wagner HL, Smith J. Facial expression in the presence of friends and strangers. J Nonverbal Behav 1991;15:201-14.

38. Botvinick M, Jha AP, Bylsma LM, Fabian SA, Solomon PE, Prkachin $\mathrm{KM}$. Viewing facial expressions of pain engages cortical areas involved in the direct experience of pain. Neuroimage 2005;25:312-9.

39. Eritz $\mathrm{H}$, Hadjistavropoulos $\mathrm{T}$. Do informal caregivers consider non-verbal behavior when they assess pain in people with dementia? J Pain 2011;12:331-9.

40. Craig KD. Social communication of pain enhances protective functions: A comment on Deyo, Prkachin and Mercer. Pain 2004;107:5-6.

41. Craig KD. The social communication model of pain. Can Psychol 2009;50:22-32.

42. Fillingim RB, King CD, Ribeiro-Dasilva MC, Rahim-Williams B, Riley JL. Sex, gender and pain: A review of recent clinical and experimental findings. J Pain 2009;10:447-85.

43. Chambers CT, Cassidy KL, McGrath, PJ, Gilbert CA, Craig KD. Child Facial Coding System. Revised Manual. Halifax: Dalhousie University, 1996.

44. Honoré J, Hénon H, Naveteur J. Influence of eye orientation on pain as a function of anxiety. Pain 1995;63:213-8. 


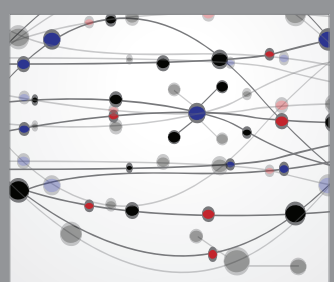

The Scientific World Journal
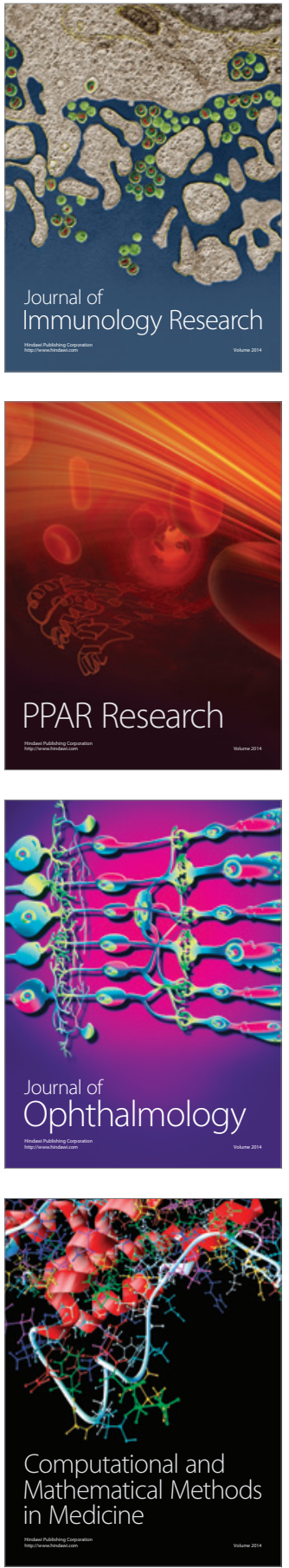

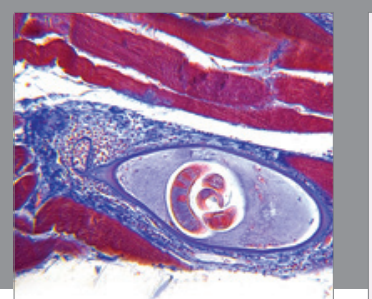

Gastroenterology Research and Practice

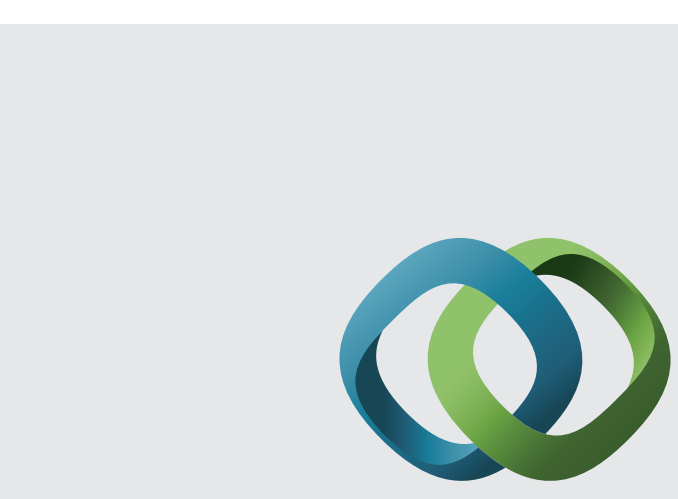

\section{Hindawi}

Submit your manuscripts at

http://www.hindawi.com
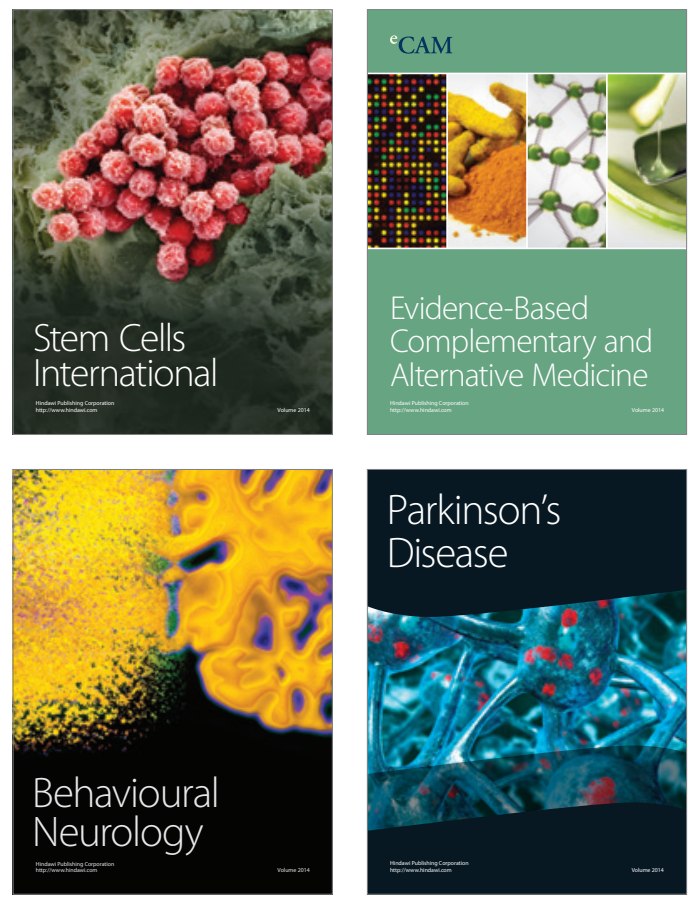
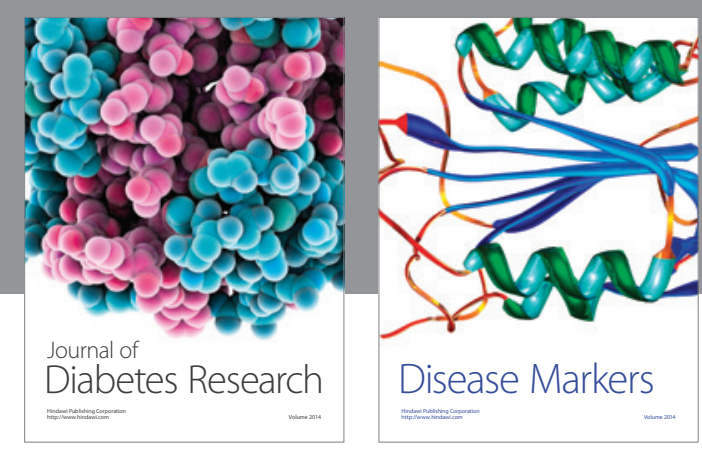

Disease Markers
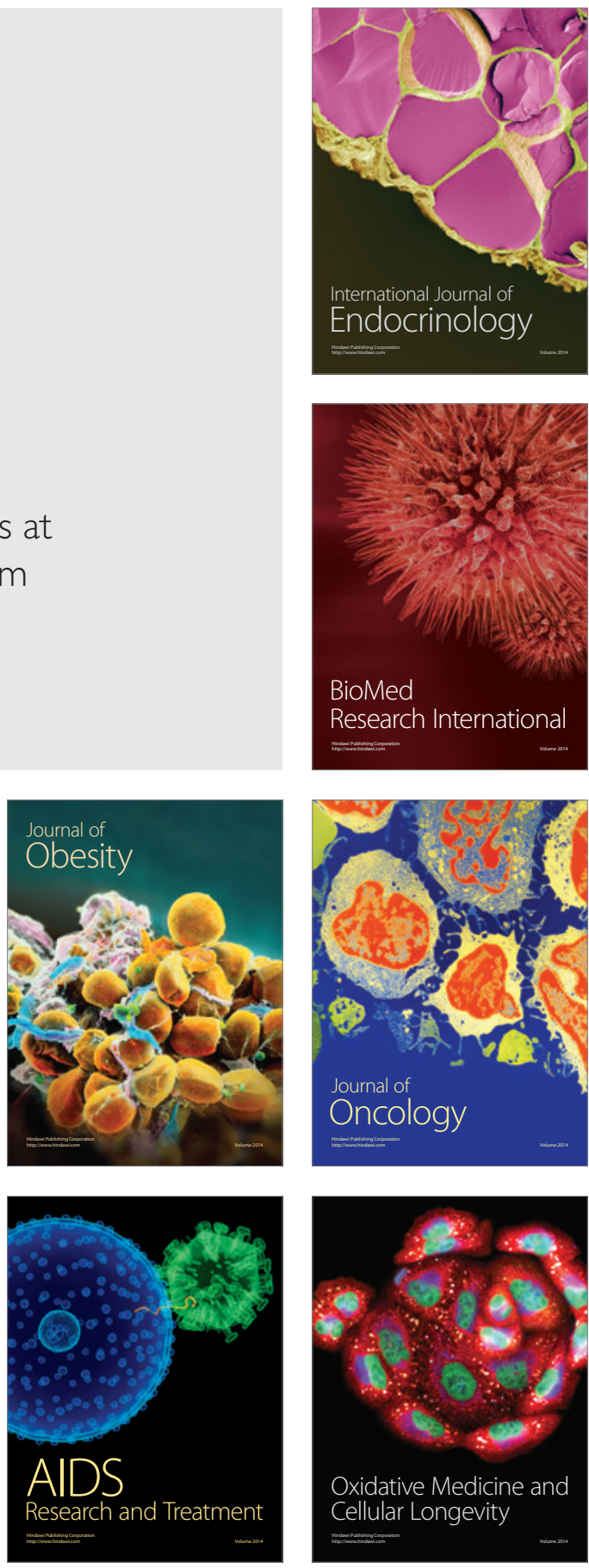